\title{
Special issue on data processing techniques and applications for Cyber-Physical Systems (DPTA 2019)
}

\author{
Chuanchao Huang ${ }^{1} \cdot$ Yu-Wei Chan ${ }^{2} \cdot$ Neil Y. Yen ${ }^{3}$
}

Published online: 11 January 2021

(C) The Author(s), under exclusive licence to Springer-Verlag London Ltd. part of Springer Nature 2021

This special issue covers cutting-edge and advanced research on data processing techniques and applications for Cyber-Physical Systems. Gathering the selected papers from the proceedings of the International Conference on Data Processing Techniques and Applications for CyberPhysical Systems (DPTA 2019), held in Shanghai, China, on November 15-16, 2019, it examines a wide range of topics, including: distributed processing for sensor data in CPS networks; approximate reasoning and pattern recognition for CPS networks; data platforms for efficient integration with CPS networks; and data security and privacy in CPS networks. Outlining promising future research directions, the special issue offers a valuable resource for students, researchers and professionals alike, while also providing a useful reference guide for newcomers to the field.

The submitted manuscripts were reviewed by experts from both academia and industry. After two rounds of reviewing, the highest-quality manuscripts were accepted for this special issue. This special issue will be published by Neural Computing and Applications as special issues. Totally, 23 papers (some are selected from DPTA 2019 conference and some are from open call) are suggested to EiC for acceptance. The selected papers are summarized as follows.

Guo and Ye [1] propose a data correctness verification scheme to verify the correctness of cloud storage data. Song et al. [2] aim at representing a more efficient and

Chuanchao Huang

carlos_huang@alumni.hust.edu.cn

1 Department of Strategic Planning and Implementation, China Merchants Bank Tower, No. 7088, Shennan Boulevard, Shenzhen 518040, China

2 Providence University, 200, Sec. 7, Taiwan Boulevard, Shalu Dist., Taichung City 43301, Taiwan, ROC

3 The University of Aizu, Aizu-Wakamatsu, Fukushima Pref. 965-8580, Japan accurate model to predict academic performance of CFCRS students which will be based on graph convolutional network. The backpropagation (BP) artificial neural network (ANN) was used by Wang et al. [3] to build a PM2.5 prediction model with the meteorological factors as input, and the predicted PM2.5 values were compared with the measured ones. The affecting the safe operation of the hoisting machinery hazards, summary and analysis based on the intrinsic safety theory and correlation analysis method, on the nature of the hoisting machinery safety assessment model is established by Chen [4]. Huang et al. [5] propose a study on the application of GIS-based artificial neural network models in the geological disaster risk zoning. The integrated use of immunology theory, complex adaptive system theory, and computational experiment technology is proposed by Yang and Yang [6] to develop an Internet network layer security detection model based on an artificial immune system as an improvement over existing models of Internet network layer security. A model about the spread of information on emergencies is constructed by Zeng et al. [7] combining empirical data and simulation experiments. The research on the optical damage of terminal optical components focuses on the search for damage points [8]. Chen et al. [9] establish a statistical model by establishing a multiple regression model to extract financial indicators that have significant effects on the financing trade of listed companies. Zhang et al. [10] propose a method that contains six individual methods, and each individual method has its own usage.

$\mathrm{Li}$ and $\mathrm{Xu}$ [11] use machine learning theory to design a variety of pattern classifiers, including Adaboost, SVM, RF, and SVR algorithms, to classify vehicles. Gao [12] builds a combined performance evaluation model based on BP neural network and rough set. Liu et al. [13] explore a Bayesian neural network-based model to predict the paths toward the realization of patent valuation. In the model, a function-effect-based patent representation. Zhen and Yao [14] take a high-tech park as an example to comprehensively evaluate the innovation ability of the high-tech park. 
Yang and Huang [15] propose a method of damage identification of prestressed concrete girder bridge based on convolutional neural network, which realizes the intelligent identification of bridge damage. Pu et al. [16] introduce the related concepts of the knowledge representation and analyze knowledge representation of knowledge graphs by category, which includes some classical general knowledge graphs and several typical domain knowledge graphs. Gao [17] uses advanced algorithms based on neural network integration and calls different algorithms: QPSO-BPNN, QPSO, PSO, PSO-BPNN, and BPNN. Han and Wang [18] use BP neural network to build a prediction model to study the analysis of inventory demand and explore the complex relationship between inventory demand and each influencing factor by training the data of inventory demand influencing factors to obtain effective measures for inventory management and control. The particle swarm optimization (PSO) algorithm is used by $\mathrm{Su}$ et al. [19] to optimize the hybrid assembly sequence planning and assembly line balance problems. The machine learning method is applied by Wang et al. [20] to the prediction of TBM penetration rate, and the established empirical model has higher accuracy and practicability. A monitoring method of volcanic ash cloud using feature fusion, convolutional neural networks (CNN) and long short-term memory (LSTM) (FF-CNN-LSTM) is presented by $\mathrm{Li}$ et al. [21]. Sun et al. [22] design a human pose recognition algorithm based on a convolutional neural network, optimize the structure and parameters of the convolutional neural network, and design a motion management system based on this algorithm. A multi-parameter (MP) IOT online measurement system based on BP NNA is designed by Liu et al. [23].

Acknowledgements The guest editors would like to thank Prof. John MacIntyre who is the editor in chief of Neural Computing and Applications. His help and trust is the most important thing for the success of this SI. The guest editors would like to thank the reviewers for their high-quality reviews, which provided insightful and constructive feedback to the authors of the papers. The guest editors also would like to thank journal editor Rachel Moriarty, Deepika Sureshkumar, and Rashmi Jenna for their help on submission and publication.

\section{References}

1. Guo Z, Ye J (2020) Improved algorithm for management of outsourced database. Neural Comput Appl. https://doi.org/10. 1007/s00521-020-05047-7

2. Hai-tao P, Ming-qu F, Hong-bin Z et al (2020) Predicting academic performance of students in Chinese-foreign cooperation in running schools with graph convolutional network. Neural Comput Appl. https://doi.org/10.1007/s00521-020-05045-9

3. Wang X, Yuan J, Wang B (2020) Prediction and analysis of PM2.5 in Fuling District of Chongqing by artificial neural network. Neural Comput Appl. https://doi.org/10.1007/s00521020-04962-z

4. Chen F (2020) Safety evaluation method of hoisting machinery based on neural network. Neural Comput Appl. https://doi.org/10. 1007/s00521-020-04963-y

5. Tan Q, Huang Y, Hu J et al (2020) Application of artificial neural network model based on GIS in geological hazard zoning. Neural Comput Appl. https://doi.org/10.1007/s00521-020-04987-4

6. Yang B, Yang M (2020) Data-driven network layer security detection model and simulation for the Internet of Things based on an artificial immune system. Neural Comput Appl. https://doi. org/10.1007/s00521-020-05049-5

7. Zhao J, Zeng D, Qin J et al (2020) Simulation and modeling of microblog-based spread of public opinions on emergencies. Neural Comput Appl. https://doi.org/10.1007/s00521-020-049192

8. Yin X (2020) Driven by machine learning to intelligent damage recognition of terminal optical components. Neural Comput Appl. https://doi.org/10.1007/s00521-020-05051-x

9. Wei X, Chen W, Li X (2020) Exploring the financial indicators to improve the pattern recognition of economic data based on machine learning. Neural Comput Appl. https://doi.org/10.1007/ s00521-020-05094-0

10. Zhang H, Yang Y, Zhang Y et al (2020) A combined model based on SSA, neural networks, and LSSVM for short-term electric load and price forecasting. Neural Comput Appl. https://doi.org/ 10.1007/s00521-020-05113-0

11. Li C, Xu P (2020) Application on traffic flow prediction of machine learning in intelligent transportation. Neural Comput Appl. https://doi.org/10.1007/s00521-020-05002-6

12. Gao J (2020) Performance evaluation of manufacturing collaborative logistics based on BP neural network and rough set. Neural Comput Appl. https://doi.org/10.1007/s00521-020-05099-9

13. Liu W, Qiao W, Liu X (2020) Discovering the realistic paths towards the realization of patent valuation from technical perspectives: defense, implementation or transfer. Neural Comput Appl. https://doi.org/10.1007/s00521-020-04964-x

14. Zhen Z, Yao Y (2020) Optimizing deep learning and neural network to explore enterprise technology innovation model. Neural Comput Appl. https://doi.org/10.1007/s00521-020-05106$\mathrm{Z}$

15. Yang S, Huang Y (2020) Damage identification method of prestressed concrete beam bridge based on convolutional neural network. Neural Comput Appl. https://doi.org/10.1007/s00521020-05052-w

16. Lin J, Zhao Y, Huang W et al (2020) Domain knowledge graphbased research progress of knowledge representation. Neural Comput Appl. https://doi.org/10.1007/s00521-020-05057-5

17. Gao Y (2020) Forecast model of perceived demand of museum tourists based on neural network integration. Neural Comput Appl. https://doi.org/10.1007/s00521-020-05012-4

18. Han C, Wang Q (2020) Research on commercial logistics inventory forecasting system based on neural network. Neural Comput Appl. https://doi.org/10.1007/s00521-020-05090-4

19. Su Y, Mao H, Tang X (2020) Algorithms for solving assembly sequence planning problems. Neural Comput Appl. https://doi. org/10.1007/s00521-020-05048-6

20. Wei M, Wang Z, Wang X et al (2020) Prediction of TBM penetration rate based on Monte Carlo-BP neural network. Neural Comput Appl. https://doi.org/10.1007/s00521-020-04993-6

21. Liu L, Li C, Sun X et al (2020) Monitoring of volcanic ash cloud from heterogeneous data using feature fusion and convolutional neural networks-long short-term memory. Neural Comput Appl. https://doi.org/10.1007/s00521-020-05050-y 
22. Li T, Sun J, Wang L (2020) An intelligent optimization method of motion management system based on BP neural network. Neural Comput Appl

23. Wang P, Liu X, Han Z (2020) Multi-parameter online optimization algorithm of BP neural network algorithm in Internet of
Things service. Neural Comput Appl. https://doi.org/10.1007/ s00521-020-04913-8

Publisher's Note Springer Nature remains neutral with regard to jurisdictional claims in published maps and institutional affiliations. 